\title{
Teil E: Fortbestand, Verwertung und Rechtsnachfolge einer BMB
}

E.1. Nachhaltigkeit und Bestandssicherung einer BMB

E.2. Welche Rolle spielt die Finanzierung einer BMB für deren Bestandssicherung? 106

E.3. Welche Regelungen und Anforderungen bestehen im Falle der Insolvenz einer BMB?

E.4. Wer ist Rechtsnachfolger einer BMB? 



\section{E.1. Nachhaltigkeit und Bestandssicherung einer BMB}

Besondere rechtliche Anforderungen an die Nachhaltigkeit und Sicherung des Bestandes einer BMB bestehen nicht. Es existieren insoweit keine spezialgesetzlichen Regelungen. Für den Bestand einer BMB ist jedoch unter Umständen die gewählte Rechtsform entscheidend. In diesem Zusammenhang ist hervorzuheben, dass z.B. ein öffentlich-rechtlicher Träger nicht insolvent werden kann. Unter dem Aspekt der Nachhaltigkeit dürfte also die öffentlich-rechtliche Trägerschaft die sicherste Rechtsform für eine BMB sein. Zudem lässt sich den Problemen aus der Insolvenz einer BMB dadurch vorbeugen, dass in der Satzung verfügt wird, wie im Falle der Zahlungsunfähigkeit mit dem Proben- und Datenbestand der BMB zu verfahren ist (z.B. der Übergang in staatliche Obhut oder in eine andere BMB).

\section{E.1.1. Datenbestand}

Der Datenbestand einer BMB unterliegt unabhängig vom Bestand der Einrichtung den Bestimmungen des einschlägigen Datenschutzgesetzes. Egal ob die Einrichtung freiwillig oder unfreiwillig liquidiert wird, müssen die verantwortlichen Personen (d.h. der Insolvenzverwalter, der Inhaber oder der Liquidator) die datenschutzrechtlichen Bestimmungen beachten.

\section{E.1.1.1. Ärztliche Daten}

Für Daten, die von einem Arzt z. B. im Zusammenhang mit der Entnahme der

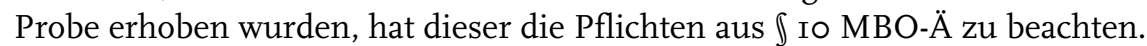
Aufzeichnungen auf elektronischen Datenträgern oder anderen Speichermedien bedürfen grundsätzlich besonderer Sicherungs- und Schutzmaßnahmen, um deren Veränderung, Vernichtung oder unrechtmäßige Verwendung zu verhindern. Gibt der Arzt seine Praxis freiwillig auf, hat er nach $\int$ Io Abs. 4 MBO-Ä dafür Sorge zu tragen, dass die Patientendaten gemäß \Io Abs. 3 MBO-Ä weiterhin mindestens für so Jahre aufbewahrt und in gehörige Obhut gegeben werden. Ein Nachfolger, dem die Patientendaten in Obhut gegeben werden, hat diese unter Verschluss zu halten und darf sie nur mit Einwilligung des Patienten einsehen oder weitergeben. Diese Vorschrift gilt unmittelbar aber nur für Daten, die der Arzt in Ausübung seines Berufes erhoben oder gewonnen hat. Nach diesseitiger Ansicht besteht sie nicht, wenn der Arzt lediglich an dem bereits entnommenen Biomaterial biomedizinische Forschung betreibt. Die biomedizinische Forschung stellt keine originär ärztliche Tätigkeit dar. Sie erfolgt auch nicht, um für den Patienten eine Diagnose, eine Therapie oder ähnliches zu bestimmen. Ausnahmen sind nur in Fällen denkbar, in denen ein behandelnder Arzt an Biomaterialien seiner eigenen Patienten forscht. Aus diesem Grund erscheint es empfehlenswert, die Aufgaben von behandelndem und forschendem Arzt strikt zu trennen. 


\section{E.1.1.2. Personenbezogene Forschungsdaten}

Personenbezogene Daten, die von einer BMB zum Zwecke der Forschung gespeichert werden, unterliegen den Sicherungsmechanismen der Datenschutzgesetze. Hier ist beispielhaft $\llbracket 40 \mathrm{BDSG}$ zu nennen, wonach personenbezogene Daten, die für Zwecke der wissenschaftlichen Forschung erhoben oder gespeichert worden sind, auch nur für diese Zwecke verarbeitet oder genutzt werden dürfen. Sobald der Forschungszweck es erlaubt, sind diese Daten zu anonymisieren. Wird eine BMB liquidiert, kann ein Forschungszweck durch die BMB mithin nicht mehr erreicht werden. Aus diesem Grund ist davon auszugehen, dass im Falle der Liquidation eine Verpflichtung zur sofortigen Anonymisierung besteht. Sollen die Forschungsarbeiten von einer anderen Einrichtung fortgesetzt werden, besteht jedoch die Möglichkeit der Übermittlung der personenbezogenen Daten nach $₫ 40$ Abs. 2 BDSG. Die Übermittlung der Daten an andere als öffentliche Stellen ist aber nur zulässig, wenn sich die empfangende Stelle verpflichtet, die übermittelten Daten nur für die vereinbarten Zwecke zu nutzen und zu anonymisieren, sobald der Forschungszweck dies erlaubt. Festlegungen aus der Einwilligungserklärung gelten ohne Einschränkung weiter und sind auch von der empfangenden Einrichtung zu beachten.

Verfolgt die BMB selbst keine Forschungszwecke, ${ }^{155}$ sondern betreibt lediglich die Lagerung und Verteilung der Proben und Daten, dann wird sie im Falle ihrer Liquidation nach $₫ 30$ Abs. 3 BDSG regelmäßig verpflichtet sein, alle personenbezogenen Daten zu löschen. Nach $₫ 30$ Abs. 3 BDSG sind personenbezogene Daten zu löschen, wenn ihre Speicherung unzulässig ist. Die Zulässigkeit der Speicherung ist in \I4 und \28 BDSG geregelt, wonach die Speicherung zweckgebunden ist. Da mit der Liquidation der BMB regelmäßig auch ihr Zweck untergeht, erscheint die Löschung zwingend geboten. Allerdings besteht auch in diesem Fall grundsätzlich die Möglichkeit der Übermittlung des gesamten Daten- und Probenbestandes auf eine andere Einrichtung. Die Vorgaben der Datenschutzgesetze sind dabei zu beachten, und der Spender ist regelmäßig rechtzeitig von der Übermittlung der Daten oder einer etwaigen Gesamtrechtsnachfolge zu benachrichtigen. ${ }^{.56}$ Diese Forderung dürfte in der Praxis jedoch nicht einfach umzusetzen sein. ${ }^{157}$

\section{E.1.2. Proben}

Der Probenbestand einer BMB unterliegt keinen besonderen Sicherungsmaßnahmen. Aus den vorausgegangenen Ausführungen ist allein abzuleiten, dass auch die Proben zu anonymisieren sind, sobald die Liquidation der BMB erfolgt. Nur die anonymisierten Proben können veräußert werden, sofern sich hierfür ein Käufer findet. Etwas anderes gilt nur dann, wenn der Spender einer Weitergabe der Proben an Dritte nicht zugestimmt bzw. ausdrücklich widerspro-

155 Dies ist bei BMB gemäß hier verwendeter Definition in der Regel der Fall.

156 Gola/Schomerus (2005), BDSG, 8 Aufl., $\$ 33$ Rdnr. 13; Simitis (2003), \28, Rdnr. 88 f.

157 Im Rahmen des TP3 des BMB-Projekts der TMF wird eine Lösung für diese Problematik skizziert. Darauf sei an dieser Stelle verwiesen. 
chen hat. Ist eine Weitergabe der Proben nicht möglich, müssen diese nach den abfallrechtlichen Regelungen entsorgt werden.

\section{E.1.3. Organisatorische Sicherungsmaßnahmen}

In diesem Abschnitt soll der Frage nachgegangen werden, ob sich durch geeignete organisatorische Sicherungsmaßnahmen verhindern lässt, dass der Proben- und Datenbestand einer BMB im Falle ihres Untergangs vernichtet bzw. anonymisiert werden muss.

\section{E.1.3.1. Auslagerung von Proben- und Datenbestand}

Eine organisatorische Sicherung gegen die Vernichtung des Proben- und Datenbestandes kann ${ }^{158}$

„namentlich dadurch geschehen, dass die BMB (als Prozess ökonomischer Wertschöpfung betrachtet) zumindest in zwei gesellschaftsrechtlich selbständige Einheiten aufgespaltet wird, so etwa in eine verwaltende Einheit, die treuhänderisch Proben und Daten ${ }^{159}$ verwaltet (etwa als BMB-Treuhand und Verwaltungs-GmbH) und eine andere, die als Erwerbsbetrieb von dieser Gesellschaft befristet Nutzungsrechte an Proben und Daten erwirbt und mit diesen Gewinne erwirtschaftet (etwa als BMB-Vertriebs $\mathrm{GmbH}$ )“.

Eine solche Probenverwaltungsgesellschaft würde regelmäßig kaum wirtschaftlichen Risiken unterliegen, so dass eine zwangsweise Liquidation in Form einer Insolvenz regelmäßig nicht vorkommen dürfte. Die Materialsammlung wäre auf diese Weise vor der Insolvenz der Vertriebs-GmbH geschützt. Dies gilt allerdings nur unter der Voraussetzung,

„dass nicht tatsächliche Verhältnisse bestehen, die den ökonomischen Zusammenhang aus Treuhand und Vertrieb rechtlich zu einem faktischen Konzern machen und damit Durchgriffshaftungstatbestände entstehen ". ${ }^{160}$

Dem ließe sich wiederum unter den Voraussetzungen des Aktiengesetzes ohne weiteres begegnen, indem es nicht zum Abschluss eines Beherrschungsvertrages kommt (『29I I I.Alt. I i.V.m. \I8 I 2 AktG.), der der Obergesellschaft ein Weisungsrecht verschafft und die unwiderlegliche Vermutung begründet, dass ein Konzern vorliegt ( $\mathbb{1}$ I8 I 2 AktG).

Den hier geschilderten Problemen ließe sich in jedem Fall dadurch begegnen, dass de lege ferenda eine öffentlich-rechtliche Organisation als Träger sämtlicher Biomaterialsammlungen bzw. aufgegebener BMB geschaffen wird. Für sie wäre das Risiko einer Insolvenz faktisch ausgeschlossen. Die Gründung einer solchen öffentlich-rechtlichen Probenverwaltungseinrichtung hätte zu-

$158 \mathrm{Vgl}$. Goebel/Evaluation (2005), S. 15.

159 Für Daten ist $\oint 30$ BDSG zu beachten.

160 Ebenda, Anm. 158. 
Teil E: Fortbestand, Verwertung und Rechtsnachfolge einer BMB

dem den Vorteil der Gewährleistung der Kontinuität sämtlicher existierender Sammlungen.

\section{E.1.3.2. Zweckbestimmung in der Satzung}

Für den Fall der freiwilligen Liquidation kann ähnlich wie in Vereinssatzungen von gemeinnützigen Vereinen bereits im Gesellschaftsvertrag oder in der Satzung eine Regelung aufgenommen werden, auf wen das Vermögen der BMB einschließlich der Proben und des Datenbestandes übergehen soll. Dies ist allerdings nur zulässig (vgl. \40 BDSG), wenn der Proben- und Datenbestand auf eine Einrichtung übergeht, die wissenschaftliche Forschung oder einen identischen Zweck wie die zu liquidierende Einrichtung betreibt.

\section{E.2. Welche Rolle spielt die Finanzierung einer BMB für deren Bestandssicherung?}

Die Finanzierung einer BMB spielt grundsätzlich keine Rolle für deren Bestandssicherung. Nur im Fall einer (nach diesseitiger Ansicht unter bestimmten Voraussetzungen erlaubten) Sicherungsübereignung von anonymisierten Proben auf einen Geldgeber kommt die Finanzierung zum Tragen, da dann nicht einmal die anonymisierten Proben durch Dritte verwertet werden können. Der Sicherungsgeber hat stattdessen ein Absonderungsrecht und damit einen Herausgabeanspruch. Im Falle der öffentlich-rechtlichen Förderung sehen einige Richtlinien zudem vor, dass dem Mittelgeber Verwertungsrechte zustehen. Diese Verwertungsrechte werden regelmäßig aber nur an den Forschungsergebnissen, nicht aber an den Proben oder Daten begründet.

\section{E.3. Welche Regelungen und Anforderungen bestehen im Falle der Insolvenz einer BMB?}

Eine zentrale Frage, der insbesondere in der anglo-amerikanischen Literatur nachgegangen wird, betrifft den rechtlich einwandfreien Umgang mit Proben und Daten im Fall der Insolvenz. ${ }^{161}$

\section{E.3.1. Probe im Eigentum des Spenders}

Wenn die Eigentumsrechte an den Proben, wie in Deutschland in der Praxis üblich, von den Spendern nicht auf die BMB übertragen werden, dann kann

161 Bei international organisierten BMB richtet sich das Verfahren im Falle der Insolvenz nach dem Recht des Landes, in dem die von der Insolvenz betroffene BMB gelegen ist. Ist nicht nur diese BMB von der Insolvenz betroffen, z.B. wenn sie Teil eines internationalen Verbunds von BMB ist, dann richtet sich das Verfahren in Europa danach, in welchem Land das Insolvenzverfahren eröffnet wurde. 
der Spender in Wahrnehmung seiner Eigentumsrechte entweder die Herausgabe der Proben verlangen oder der Weiterverwendung der Proben z.B. durch den Insolvenzverwalter zustimmen. Kommt es hingegen zur Liquidation, muss der Spender sich entscheiden, ob er die Vernichtung oder die Herausgabe der Materialien wünscht. Sofern eine entsprechende Möglichkeit besteht, könnte der Spender dem Insolvenzverwalter gegenüber auch erklären, dass die Proben an eine andere BMB oder Forschungseinrichtung abzugeben sind. Der Spender ist in jedem Fall zwingend vom Insolvenzverwalter über die Insolvenz der BMB zu unterrichten. Dies ergibt sich bereits aus der Insolvenzordnung, da er als Eigentümer der Probe Gläubiger der Gemeinschuldnerin ist und etwaige Rechte anmelden könnte. Meldet er seine Rechte an den Proben nicht innerhalb der gesetzten Fristen der Insolvenzordnung an, kann der Insolvenzverwalter diese vernichten.

Auch wenn an der Probe nur ein Nießbrauch eingeräumt wurde, besteht ein Pfändungs- und Insolvenzschutz. Ein Nießbrauchsrecht ist nämlich weder übertragbar noch veräußerlich. Nach \85I I ZPO ist eine Forderung aber nur der Pfändung unterworfen, wenn sie übertragbar ist. Als Ausnahme wäre ein unveräußerliches Recht gemäß \857 Abs. 3 ZPO in Ermangelung besonderer Vorschriften insoweit pfändbar, als die Ausübung einem anderen überlassen werden kann, was beim Nießbrauch eines Biomaterials aus vielen Gründen aber nur eingeschränkt oder gar nicht der Fall sein dürfte.

\section{E.3.2. Probe im Eigentum der BMB}

Stehen die Proben im Eigentum der BMB, so gelten im Falle der Insolvenz der BMB dieselben Grundsätze wie unter E.I. beschrieben. Der Insolvenzverwalter hat die datenschutzrechtlichen Bestimmungen ebenso zu beachten, wie die Verantwortlichen der BMB. Dem entsprechend hat auch der Insolvenzverwalter im Falle des Untergangs einer BMB alle personenbezogenen Daten zu löschen und die Proben zu anonymisieren. Auch für diesen Fall könnte es sinnvoll sein, entsprechende Vorkehrungen und Zweckbestimmungen für die Probenverwendung bereits in der Einwilligungserklärung festzulegen.

Fraglich ist, ob die anonymisierten Proben in die Insolvenzmasse fallen. Nach \36 InsO gehören Gegenstände, die unpfändbar sind und damit nicht der Zwangsvollstreckung unterliegen, nicht zur Insolvenzmasse. Die Unpfänd-

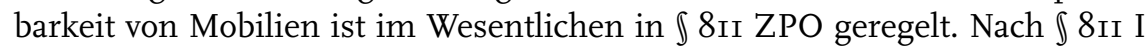
Nr. 5 ZPO sind bei Personen, die aus ihrer körperlichen oder geistigen Arbeit oder sonstigen persönlichen Leistungen ihren Erwerb ziehen, die Gegenstände unpfändbar, die zur Fortsetzung dieser Erwerbstätigkeit erforderlich sind..$^{162}$ Bei einer BMB, die in Trägerschaft einer Privatperson bzw. einer GbR oder OHG steht, wäre dies der Probenbestand.

Nicht ausdrücklich in \8II ZPO genannt, aber dennoch unpfändbar sind bestimmte Gegenstände, deren Veräußerung durch den Gerichtsvollzieher 
ausgeschlossen sind. ${ }^{163}$ Dies gilt z.B. für eine Leiche ${ }^{164}$ und für Gegenstände, die von einem Persönlichkeitsrecht überlagert sind. ${ }^{165}$ Spezialgesetzlich ist eine solche Unpfändbarkeit z.B. in $\iint$ II4, II 6 und IIg UrhG geregelt. Daraus ergibt sich, dass der personenbezogene Datenbestand, der schon keine Sache darstellt, der Pfändung nicht unterliegt und damit auch nicht in die Insolvenzmasse fällt. Die Proben sind zwar im rechtstechnischen Sinne nicht mit einer Leiche vergleichbar, da sie nach der herrschenden Auffassung bewegliche Sachen sind. Sie sind aber vom Persönlichkeitsrecht des Spenders überlagert. Denkbar wäre danach, in entsprechender Anwendung der $\int \mathbb{\int I I 4}$, II 6 und II9 UrhG bzw. $\ 22$ KurhG, die Zwangsvollstreckung nur nach Zustimmung des Spenders zu gestatten. Andererseits wird das allgemeine Persönlichkeitsrecht des Spenders umso schwächer, je geringer der Personenbezug ausfällt. Eine Pfändung anonymisierter Proben käme demnach zwar in Betracht, eine freie Versteigerung und Veräußerung an jeden Dritten dürfte aber mit Sicherheit nicht möglich sein.

Unpfändbarkeit besteht auch für zweckgebundene Forderungen, es sei denn, sie würden trotz der Pfändung einer zweckentsprechenden Verwendung zugeführt. Proben, die nur zum Zwecke der nicht-kommerziellen Forschung, nur für ein bestimmtes Forschungsprojekt oder unter dem Vorbehalt der Nichtübertragbarkeit zur Verfügung gestellt bzw. übereignet wurden, dürften daher auch nur für diesen Zweck gepfändet werden. ${ }^{166}$ Der Insolvenzverwalter kann die Proben demnach auch nur insoweit der Insolvenzmasse zuführen, als sie einer zweckentsprechenden Verwendung zugeführt werden können.

Hieraus lässt sich schließen, dass die Probensammlung einer insolventen BMB nur in wenigen Fällen gepfändet bzw. zur Insolvenzmasse gezählt werden könnte. Eine Verletzung der Patientenrechte ist damit weitestgehend ausgeschlossen. Allerdings führt die Unpfändbarkeit letztlich zu dem Ergebnis, dass die Proben in den meisten Fällen vernichtet oder an die Spender herausgegeben werden müssen und somit nicht als Sicherungsmittel für einen Kredit in Frage kommen. Es erscheint daher empfehlenswert, das Eigentum an den Proben einer reinen Verwaltungsgesellschaft zu übertragen, die dann entweder Forschungseinrichtungen oder anderen BMB als Verwertungsgesellschaften gegen eine Aufwandsentschädigung bzw. ein Entgelt einen beschränkten Nießbrauch an den Proben einräumt. Bei den Nießbrauchnehmern werden die Proben dann gelagert, und diese müssen auch sämtliche für Erhalt und Lagerung der Proben anfallenden Kosten tragen.

163 Vgl. $\$ 126$ GVGA und 129 a GVGA.

164 Schilke, MüKo, $\$ 811$ ZPO, Rdnr. 57; so auch Schuschke/Walker, Zwangsvollstreckung und einstweiliger Rechtsschutz, $\$ 811$ Rdnr. 47.

165 KG OLG-Rspr. 25, 168; Münzberg/Stein-Jonas, $\$ 811$ ZPO, Rdnr. 74, 21. Aufl.

166 Münzberg/Stein-Jonas, $\$ 811$ ZPO, Rdnr. 77. 


\section{E.4. Wer ist Rechtsnachfolger einer BMB?}

Die Rechtsnachfolge einer BMB ist abhängig von der Frage, in welcher Rechtsform der ursprüngliche Träger der BMB firmiert hat. Alle weiteren Überlegungen sind rechtspolitischer Natur, denn aus den bestehenden Gesetzen ergeben sich keine Absicherungen für den Probenbestand. Zwar haben die Forschung und zum Teil auch die Spender generell ein berechtigtes Interesse am faktischen Fortbestand einer BMB. Es ist aber unklar, ob es sich bei Biomaterialien wirklich um ein so wichtiges Gut handelt, dass daran ein erhebliches Gemeinschaftsinteresse bestehen könnte, zumal wenn von Seiten der Forschung immer wieder der geringe ökonomische Wert der einzelnen Probe betont wird. Damit ist nicht ausgeschlossen, dass es sich bei Proben um ein überragendes Kulturgut handeln könnte. Allerdings lassen sich in den bisher publizierten Argumenten dafür keine Anhaltspunkte finden. Dem widerspricht auch nicht, dass das Individualrecht an der Probe als überragendes Persönlichkeitsrecht geschützt wird, denn dies ist ein Argument, das vom einzelnen Rechtsträger ausgeht und allenfalls von ihm geltend gemacht werden kann. Insofern sind Anregungen, etwa Auffanggesellschaften zur Erhaltung von Proben als wichtige Kulturgüter zu gründen, nach Ansicht der Gutachter derzeit noch $\mathrm{zu}$ weit gegriffen. 